\title{
Correção de membrana epirretiniana por meio de vitrectomia pars plana em paciente jovem: um relato de caso
}

\author{
correction of idiopathic epiretinal membrane by pars plana vitrectomy in young pacient: a \\ case report
}

\section{Corrección de la membrana epirretiniana idiopática mediante vitrectomía pars plana en paciente joven: reporte de un caso}

Gabriel Ribeiro Neiva ${ }^{1 *}$, Isabela Gomes Lima ${ }^{1}$, Tereza Cristina Moreira Kanadani ${ }^{1}$, Rodrigo dos Anjos Versiani ${ }^{2}$.

\section{RESUMO}

Objetivo: O principal objetivo do estudo é detalhar o tratamento realizado em paciente jovem com Membrana epirretiniana sua patogênese e progressão do caso. Detalhamento do caso: Paciente com perda progressiva da acuidade visual em olho esquerdo com 1 ano de evolução chegando a 20/70. Por ser um paciente jovem, 24 anos, sua patogênese se deve a um trauma que provocou o acometimento visual. Exames realizados como OCT's e mapeamentos de retina indicaram piora do quadro progressivamente. Optou-se por realizar uma vitrectomia pars-plana para retirada de membrana. Também foi realizado laser para auxílio do tratamento. O paciente teve uma melhora significativa após tratamento. Considerações finais: Sendo assim a vitrectomia pars plana foi a indicação ideal para o paciente em questão sendo o melhor tratamento para melhora da acuidade visual. Foi possível perceber também que o uso do laser de fotocoagulação pré cirúrgico e a técnica utilizada permitiram a redução das complicações e recidiva.

Palavras-chave: Membrana-epirretiniana, Vitrectomia, Retina.

\begin{abstract}
Objective: The main objective of the study is to get into the details of the treatment performed in a young patient with epiretinal membrane, its pathogenesis and progression of the case. Case details: Patient with progressive loss of visual acuity in the left eye with 1 year of evolution reaching 20/70. Being a young patient, 24 years old, its pathogenesis is due to a trauma that caused visual impairment. Exams performed as OCT's and retinal scans indicated progressive worsening of the condition. It was decided to perform a pars-plana vitrectomy to remove the membrane. Laser was also performed to aid the treatment. The patient had a significant improvement after treatment. Final considerations: Therefore, the pars plana vitrectomy was the ideal indication for the patient in question, being the best treatment to improve visual acuity. It was also possible to notice that the use of pre-surgical photocoagulation laser and the technique used allowed for the reduction of complications and recurrence.
\end{abstract}

Keywords: Epirretinal-membrane, Vitrectomy, Retina.

\section{RESUMEN}

Objetivo: El objetivo principal del estudio es a detallar el tratamiento realizado en un paciente joven con membrana epirretiniana, su patogenia y progresión del caso. Detalle del caso: Paciente con pérdida progresiva de agudeza visual en el ojo izquierdo con 1 año de evolución llegando a 20/70. De paciente joven, de 24 años, su patogenia se debe a un trauma que le provocó discapacidad visual. Los exámenes realizados, como la OCT y las exploraciones de retina, indicaron un empeoramiento progresivo de la afección. Se decidió

${ }^{1}$ Faculdade Ciências Médicas de Minas Gerais, Belo Horizonte - MG. *E-mail: gabrielribeiroca@gmail.com

2 Núcleo de Excelência em Oftalmologia (NEO), Belo Horizonte - MG.

SUBMETIDO EM: 6/2021

PUBLICADO EM: 8/2021 
realizar una vitrectomía pars-plana para remover la membrana. También se realizó láser para ayudar en el tratamiento. El paciente tuvo una mejoría significativa después del tratamiento. Consideraciones finales: Siendo así la vitrectomía pars plana fue la indicación ideal para el paciente en cuestión, siendo el mejor tratamiento para mejorar la agudeza visual. También se pudo notar que el uso de láser de fotocoagulación prequirúrgico y la técnica empleada permitieron la reducción de complicaciones y recidivas.

Palabras clave: Membrana-epirretiniana, Vitrectomía, Retina.

\section{INTRODUÇÃO}

A Membrana Epirretiniana (MER) é uma patologia caracterizada pela presença de uma estrutura celular organizada na superfície interna da retina, que pode estar localizada na macula e se estender até a porção vascular da retina sem causa específica, mas pode estar associada a processos inflamatórios crônicos intraoculares. A patogênese dessa condição depende da atividade das células retinianas como os fibroblastos, células da glia, epitélio pigmentar, processos de inflamação e cicatrização. Processos que incluem traumas oculares, inflamações intraoculares, cirurgias prévias, desprendimento posterior idiopático do vítreo além de patologias que acometam a retina aumentam a incidência da MER (JUAREZ AB, 2003).

A MER é mais frequente em pacientes com mais de 50 anos de idades estando presente em 3,5 a 5,5\% de olhos submetidos a autópsias, sendo bilaterais em 20 a 30\% dos casos e normalmente assimétricas. Quando são unilaterais, após acompanhamento por 5 anos, ocorre envolvimento do segundo olho em 13,35\% dos casos. O sexo feminino é o mais acometido, tendo prevalência na população geral de 7 a $11,8 \%$ (NOVELLI FJ, 2018).

A maioria dos pacientes são assintomáticos sendo seu diagnóstico normalmente realizado em exames oculares de rotina. Na maior parte dos pacientes a acuidade visual (AV) é boa sendo que $67 \%$ relatam visão melhor ou igual a 20/30 e apenas 5\% têm visão inferior a 20/200 (NOVELLI FJ, 2018). A classificação de AV é uma relação de proporção que indica o quão distante a pessoa está enxergando, sendo assim a melhor possível 20/20 e de acordo com a tabela de Snellen a pior seria 20/200. Quando o paciente possui queixas oculares elas são pouco precisas como borramento da visão e imagens, alteração da linearidade de imagens e distorção de objetos (JUAREZ AB, 2003).

O único tratamento definitivo estabelecido para resolução do caso é por via cirúrgica. Dessa forma, o procedimento usual da MER é a Vitrectomia Pars Plana (PPV). Essa técnica permite o acesso a retina por meio da remoção do humor vítreo e substituição por um líquido ou gás, são utilizados trocanters que entram pela esclera para esse acesso. Estudos recentes demostraram que tal cirurgia associada a uma descamação da camada de tecido da membrana acoplado a face interna da retina permite uma melhora dos sintomas em pacientes com perda da AV, grande sucesso anatômico e redução nos casos de recidiva (GOVETTO A, et al., 2019). Somado a isso, fotocoagulação prévia pode ser importante para prevenir complicações futuras (LUCENA DR, 2020).

Sendo assim, o objetivo do estudo é detalhar da forma mais clara o tratamento realizado nesse paciente, bem como o seu caso como um todo, a fim de compreender melhor essa abordagem da MER em pacientes jovens, sua progressão e avaliação clínica.

\section{DETALHAMENTO DO CASO}

Neste caso o paciente de 24 anos, míope (OD -6,0; OE -5,5) sem demais morbidades com relato de perda progressiva da AV em Olho Esquerdo (OE) com início dos sintomas em 26/07/2019. De história pregressa apresenta trauma em região temporal esquerda no dia 12/01/2019. Em primeira avaliação no dia 26/10/2019 se obteve AV de 20/40 sem demais alterações. Em segunda medida, dia 15/01/2020 obteve-se AV de 20/50. Em consulta realizada 09/07/2020 onde foram realizados exames para acompanhar a progressão da membrana. Foi realizada uma fundoscopia em que podemos perceber na Figura 1 uma retração com tangenciamento centrípeto da retina causados pela membrana principalmente em região de fóvea central, sem descolamento da mesma, formação de neovasos ou estreitamento arteriolar. 
Figura 1 - Fundoscopia realizada em 09/07/2020.

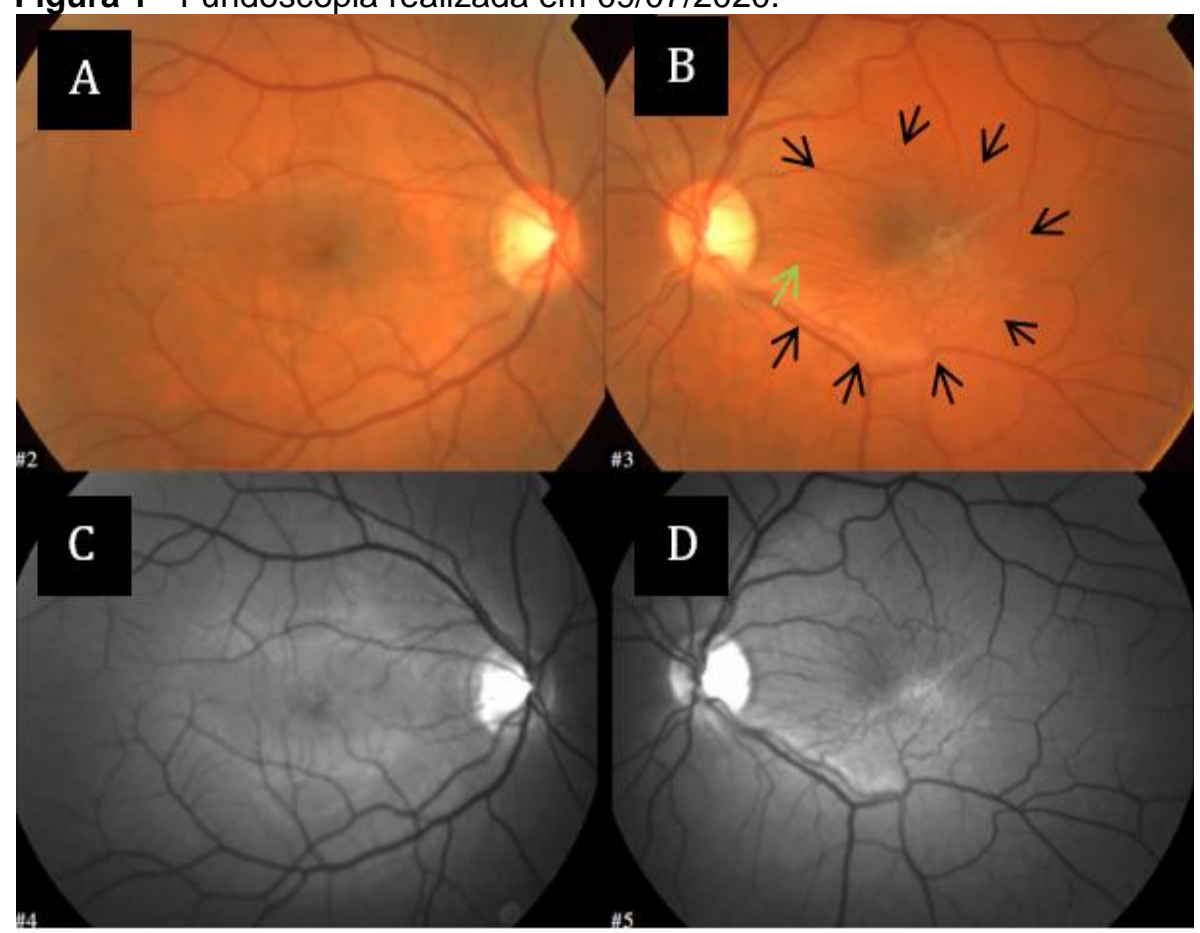

Legenda: A: Olho direito não acometido. B: Olho esquerdo acometido pela membrana. Setas pretas indicam membrana epirretiniana provocando retração com tangenciamento centrípeto da região foveal indicado pela seta verde. C: Olho direito não acometido com imagem sem coloração. D: olho esquerdo acometido com figura sem coloração. Fonte: Neiva GR, et al., 2021.

Tal quadro progrediu de forma que sua AV em última avaliação na data 27/07/2020 foi 20/70. Junto ao quadro havia relato de metamorfopsia, macropsia, moscas volantes e flashes luminosos que progrediram com o avançar do caso. Por ser um paciente jovem, acredita-se que sua patogênese pode estar relacionada a um trauma na região temporal esquerda durante realização de esporte aquático coincidente com a data dos sintomas. É possível perceber no OCT datado 27/07/2020, na Figura 2 e Figura 3, aumento da espessura da região foveal central medindo cerca de 461un definindo a membrana como única, irregular, associada a rugas subjacentes da retina e classificada de acordo com o estadiamento de Govetto A, et al. (2019), 4 graus devido ao nível de desorganização foveal. Não se observou edema macular cistóide e cotton balls.

Figura 2 - OCT de Olho Esquerdo realizado em 27/07/2020.

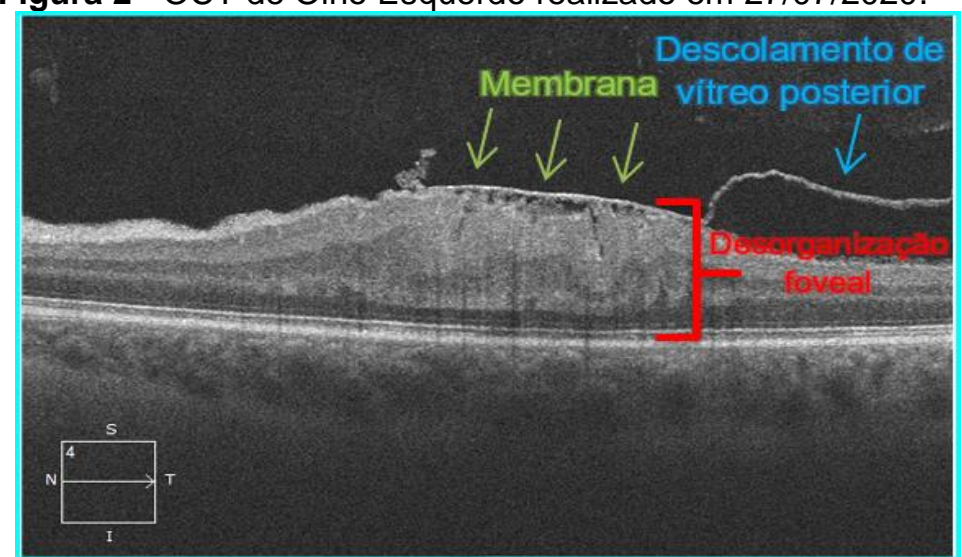

Legenda: Setas verdes: membrana na região da fóvea realizando tração na mesma. Colchete vermelho: desorganização das camadas visuais classificada como grau 4. Seta azul: provável descolamento de vítreo posterior que por sua vez foi causado pela tração realizada pela membrana na região foveal. Fonte: Neiva GR, et al., 2021. 
Figura 3 - OCT de Olho Esquerdo e Olho Direito realizado em 27/07/2020.

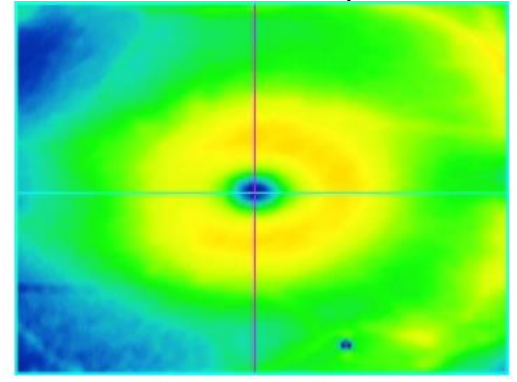

Fovea: 249, 66
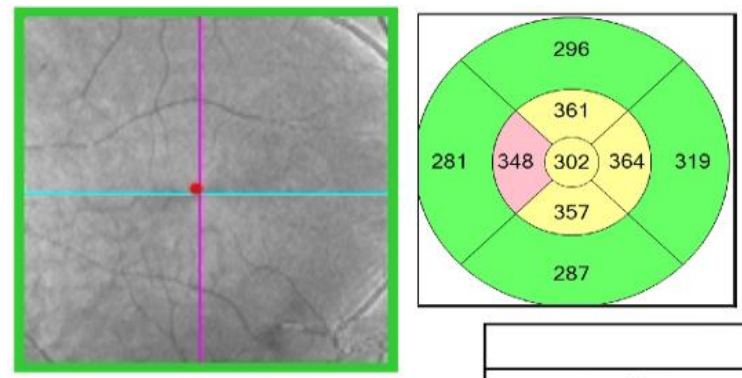

287

\begin{tabular}{|c|c|c|}
\hline & OD & OE \\
\hline Espessura central: & 302 & 553 \\
\hline Volume: & 11 & 12.8 \\
\hline Espessura media: & 306 & 356 \\
\hline
\end{tabular}
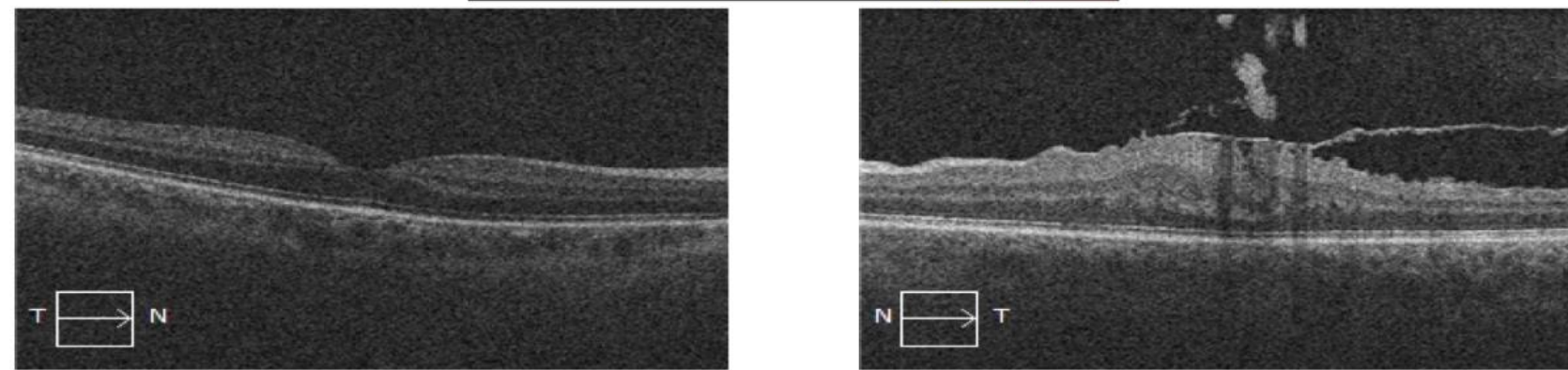

Fonte: Neiva GR, et al., 2021; imagens produzidas por: USERCIR-EOFKULQ SW Ver: 11.0.0.29946 Copyright 2018 Carl Zeiss Medietec, Inc. Todos os direitos reservados.

Para o paciente em questão optou-se por realizar uma PPV para retirada de membrana com exérese da camada limitante interna da retina. Tal retirada é relacionada a menor incidência de recidiva. Anterior ao procedimento realizou-se uma fotocoagulação periférica no fundo de olho para estabilizar a retina e prevenir futuras complicações. A PPV foi realizada no dia 13/08/2020. A Figura 4 é uma foto realizada durante a cirurgia.

Figura 4 - Fotografia realizada durante a PPV realizada em 13/08/2020.

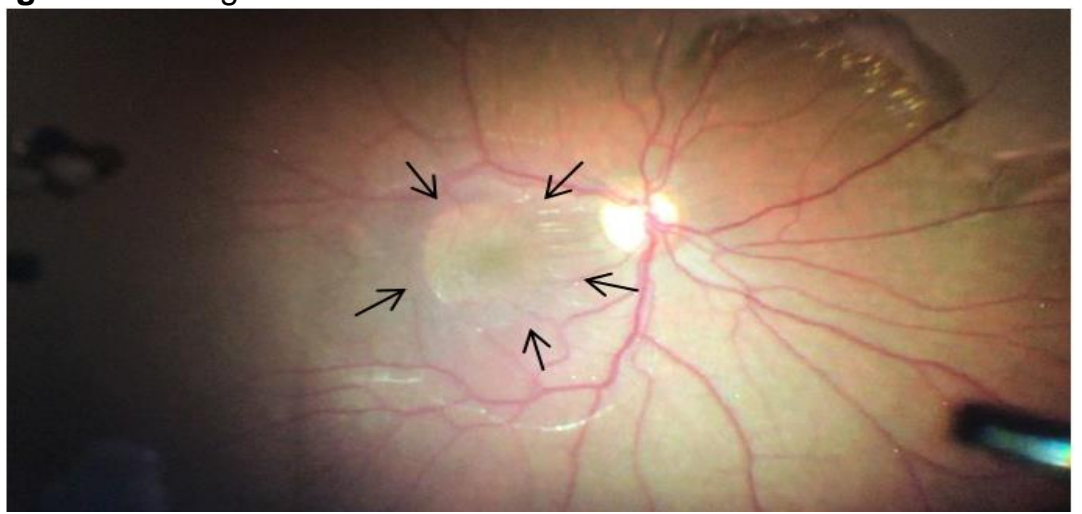

Legenda: Setas indicam região em que há a descamação da membrana realizada durante a cirurgia. Fonte: Neiva GR, et al., 2021. 
Após a cirurgia o paciente passou por consultas de acompanhamento pós cirúrgico onde foram realizados OCT's. Foram realizadas consultas pós cirúrgicas nas datas 17/08/2020, 31/08/2020, 17/09/2020 em que foi realizado o primeiro OCT pós PPV, 06/10/2020 em que foi realizado o segundo, e no dia 11/01/2021 em que foi realizado novo exame mostrado na Figura 6B. No dia 17/11/2020 foi realizada uma fundoscopia representada na Figura 5 na qual é possível observar a região foveal central ausente de membrana.

Figura 5 - Fundoscopia realizada em 17/11/2020.

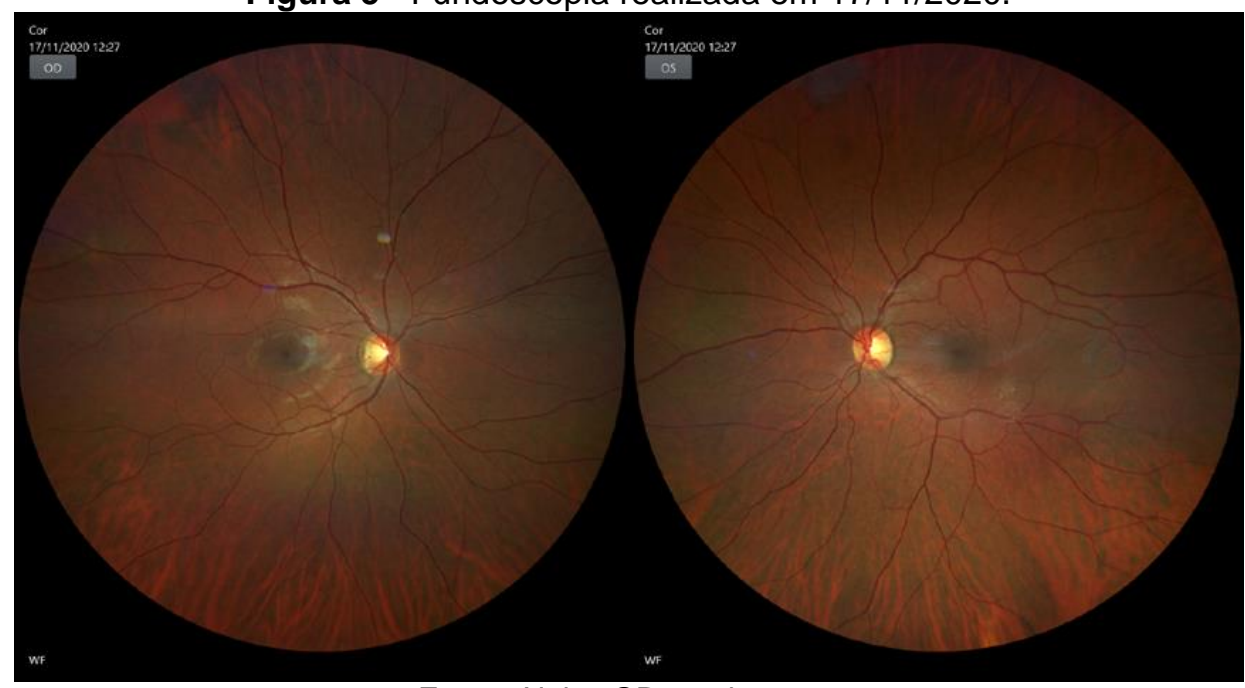

Fonte: Neiva GR, et al., 2021.

Na Figura 6A é possível observar o comparativo dos OCT's de pós-operatório imediato do paciente e tardio, datados em 06/10/2020 e 11/01/2021 em que é possível perceber que os resultados formam mantidos durante o pós operatório. Na última consulta o paciente realizou acompanhamento e OCT com imagens (Figura 6B) representadas sendo possível observar uma redução da elevação da depressão foveal insinuando uma fóvea progredindo para sua forma fisiológica.

Figura 6 - OCT`s Comparativos da data 06/10/2020 E 11/01/2021 E OCT de 11/01/2021.

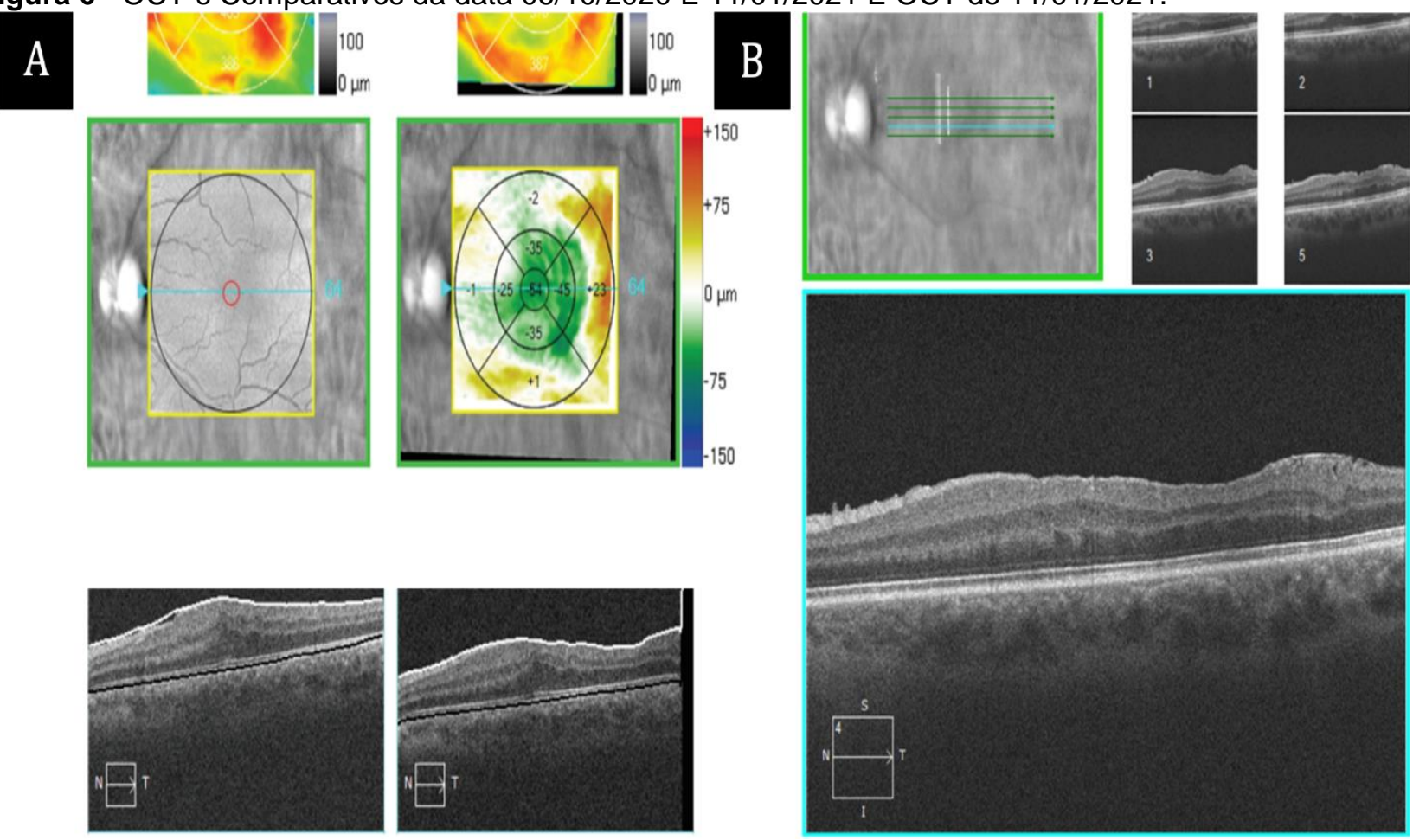

Legenda: A) Comparativo dos OCT's do dia 06/10/2020 e 11/01/2021; B) OCT do dia 11/01/2021.

Fonte: Neiva GR, et al., 2021; imagens produzidas por: USERCIR-EOFKULQ SW Ver: 11.0.0.29946 Copyright 2018 Carl Zeiss Medietec, Inc. Todos os direitos reservados. 


\section{DISCUSSÃO}

A MER se trata de uma estrutura que se forma no fundo de olho anterior e aderido a retina recobrindo a depressão foveal fisiológica da retina. Sua patogênese é pouco elucidativa, mas acredita-se estar relacionada a processos inflamatórios crônicos e degenerativos relacionados a idade ou patologia de base dentre eles descolamento de vítreo posterior, pós-operatório de descolamento de retina, patologias vasculares, hemorragia persistente e traumatismos. Sendo assim, a maioria dos pacientes com tal alteração estão acima de 60 anos ou apresentam alguma patologia que favoreça ataques contra a retina (SÁNCHEZ-VICENTE JL, et al., 2019).

Quanto ao tempo de evolução este também é variável, visto que depende de processos inflamatórios e reações próprias de cada organismo (GOVETTO A, et al., 2019). O paciente relata trauma aquático em região temporal esquerda que precedeu tais alterações visuais sendo uma das hipóteses do surgimento.

Com sua evolução a MER se altera e podem ser observadas algumas fases sendo a primeira a alteração do reflexo celofane, seguida as duas outras que são as principais causadoras de sintomas. São elas a opacidade progressiva e contração centrípeta, com tração e formação de ondulações na retina que causam uma elevação da depressão foveal reduzindo a qualidade da visão (CHEN H, et al., 2019).

O paciente relatava tais alterações que se caracterizavam por uma opacificação da visão relatada como se estivesse vendo a partir de um "papel celofane" e em data posterior relatou sintomas de metamorfopsia, macropsia e flashes luminosos que, por sua vez, são provenientes da tração que pode distorcer a retina e desordenar as linhas visuais. Relatou também presença de moscas volantes causados pelo descolamento parcial do vítreo posterior.

A resolução espontânea é rara ocorrendo em menos de $1 \%$ das vezes. Inexistem tratamentos clínicos com eficácia sendo indicada, portanto, sua retirada via cirúrgica a única forma definitiva de resolver o problema (ARAMBURU GG e ARANA AL, 2005). Existem fatores prognósticos positivos importantes que se relacionam a AV pós cirúrgica dentre eles edema macular cistóide, "cotton balls" que representam elevação e tração dos fotorreceptores e tempo prolongado de evolução, todos relacionados a um pior resultado final (JUAREZ AB, 2003). No caso em questão, a intervenção antes do surgimento desses fatores se fez importante.

Como a análise das alterações externas da retina podem ser insuficientes para prever o resultado visual pós-operatório, a atenção voltou-se para o estudo da retina interna, o principal local afetado pelo estresse mecânico da MER. A presença de camadas foveais internas ectópicas contínuas (CFIEC) na região foveal central foi recentemente descrita com um OCT em olhos com MER idiopáticas.

A formação da CFIEC pode representar um sinal importante a progressão da MER e é peça central no esquema de estadiamento recentemente proposto para tais de Govetto A, et al. (2019), e assim podemos estadiar a membrana epirretiniana em quatro níveis de acordo com o grau de acometimento do paciente, que neste caso se encontra em estadiamento grau 4.

Segundo Govetto A, et al. (2019), o Estágio 1 é a MER leve com poucas modificações anatômicas nas linhas visuais, depressão foveal mantida, camadas da retina bem definidas. Quando progredimos para o Estágio 2 percebemos membranas com alterações mais avançadas, perda da depressão foveal, porém as camadas da retina ainda se encontram bem definidas. Progredindo ainda mais encontramos o Estágio 3 , CFIEC recobrem toda a fóvea, matem-se a perda da depressão foveal, e as camadas da retina ainda se apresentam mantidas e como último grau de progressão temos Estágio 4, que é o que o paciente se encontra, pois, sua MER está com avançado grau de desorganização foveal, CFIEC mantem recobrindo toda a área foveal, mantem-se a perda da depressão foveal, porém as camadas da retina são interrompidas (GOVETTO A et al, 2019).

A indicação cirúrgica se baseia em alguns pilares sendo o mais importante o sintoma e a AV que o paciente apresenta, visto que em diversos casos não se pode fazer uma relação direta entre estrutura anatômica da retina e funcional. Dessa forma a $\mathrm{AV}$ seria um forte indicador considerando importante avaliação cirúrgica para uma AV menor ou igual 20/60. Além disso, a presença de alguns sintomas específicos como 
metamorfopsia e macropsia são definidores para a escolha do tratamento pois evidenciam danos avançados de acometimento da retina. Outras alterações importantes para a definição do tratamento cirúrgico, mas que não foram observadas no caso são a presença de edema macular cistóide, cotton balls, neovasos e descolamentos (GOVETTO A, et al., 2019). A cirurgia indicada e que foi realizada pelo paciente em questão se trata da PPV.

A PPV é um procedimento cirúrgico que consiste na remoção do humor vítreo a fim de obter melhor acesso a retina. Isso é usado para o tratamento de diversas condições como retinopatia da prematuridade, retinopatia diabética, remoção de corpos estranhos intraoculares degeneração macular entre outros problemas da câmara anterior e uveítes emoção de tecido cicatricial, uso de laser para descolamento de retina e tratamento de buraco macular (STAVROU P, et al., 2001; YU Y, et al., 2017). O paciente do caso foi submetido a PPV para permitir acesso a retina para a remoção da membrana epirretiniana.

Antes de realizar o procedimento existem autores que relataram benefícios ao realizar fotocoagulação na periferia da retina pré-cirúrgica (LUCENA DR, 2020). A fotocoagulação é um laser muito utilizado nos tratamentos da retina, tais como obstruções vasculares da retina, retinopatia diabética, dentre outros (MARQUES MF, et al., 2017; SOUSA JJAS, 2018). No paciente o laser foi feito com intuito de bloquear áreas de degeneração periférica da retina em risco de evoluir para descolamento. Para isso foi feito o procedimento sendo uma prevenção frente a intervenção por qual a que a retina vai passar.

Após o acesso é utilizada uma solução de maior densidade que o humor vítreo que pode ser salina, óleo siliconado ou algum gás para preencher o local onde foi removido a fim de manter a retina na posição correta (YANNUZZI NA, et al., 2018). Usualmente, em pacientes com acometimentos retinianos como descolamento de retina o importante é que a retina se mantenha em posição correta e o mais estável possível, por isso é utilizado um gás de lenta absorção que permanece no olho do paciente por mais tempo mantendo a retina na posição correta. Entretanto esse gás pode ser tóxico ao cristalino. Apesar de raros, existem relatos que relacionam a vitrectomia ao surgimento posterior de catarata devido ao íntimo contato com esse gás (JANG $\mathrm{K}$, et al., 2021).

Sabendo disso, no paciente em questão, foi utilizado um ar de absorção rápida, isso porque a retina do paciente não apresentava nenhuma patologia que justificasse o contato por tanto tempo com a substância reduzindo a toxicidade ao cristalino.

Para o acesso ao humor vítreo é realizada a inserção de trocanteres na esclera com o olho já dilatado (TAKAHASHI VKL, 2019). Atualmente existe uma corrente que indica um melhor prognóstico e menor recidiva com o uso de trocanteres menores e menos espessos e também ao uso de micro incisões transconjutivas sem uso de suturas que possibilitam menor dor e inflamação pós-operatória, melhora no astigmatismo pósoperatório e melhora mais rápida do retorno a visão (CHATZIRALLI I, 2019; ISHIDA Y, et al., 2020; KELLNER L, et al., 2007; STEVE C, et al., 2020).

No paciente foram utilizados três trocanteres sendo o primeiro com a luz permitindo a melhor visualização da retina, o segundo com a pinça responsável pela remoção da membrana, e um terceiro que é uma espécie de dreno para a remoção do fluido do humor vítreo permitindo então esse acesso a retina (CHEN H, et al., 2019). No procedimento é utilizado um corante para facilitar a visualização da membrana e no caso em questão foi utilizado um de coloração azul que é inserido no espaço onde é removido o humor vítreo.

Após a cirurgia, os dias de recuperação são muito importantes para garantir os resultados anatômicos e funcionais da retina. Dessa forma, se faz necessário repouso e abstenção de qualquer atividade física por cerca de 1 mês, como foi feito para o paciente em questão, a variar com o caso, e dos demais afazeres como trabalho e compromissos por 15 dias o que também foi realizado. Nesse período, uma recomendação importante é que o paciente permaneça com a cabeça virada para baixo e durma em decúbito ventral (SERRANO T, 2017). Isso se deve a um maior contato do ar inserido com a retina que por ser mais denso pode ajudar a aplainar a retina sendo uma força tracional contra as ondulações formadas pela membrana. Além disso, tal posição reduz o contato da substância com o cristalino reduzindo a agressão tóxica. Com o tempo, o corpo produz uma substância com características similares ao humor vítreo que substituem o ar. 
Sendo assim, é possível perceber que a MER acomete na maioria dos casos pacientes idosos ou com patologias específicas pré-existentes, mas apesar de raro pode ser encontrada em um paciente jovem e hígido como o caso em questão. Além disso, o diagnóstico via OCT e tratamento precoce via PPV se faz importante e é uma maneira de reduzir os malefícios causados a retina, bem como recuperar uma acuidade e qualidade de visão próxima da anterior a patologia. A importância de um pós cirúrgico bem-feito também é essencial para o sucesso do procedimento como um todo. Além disso, a fotocoagulação pré-cirúrgica e retirada MLI durante o procedimento foram medidas importantes para reduzir complicações e recidiva. Dessa forma, o paciente em questão apresentou um prognóstico positivo pós a resolução cirúrgica. Esse estudo elucida a respeito do tratamento da MER em pacientes jovens sendo de grande contribuição para a oftalmologia.

\section{REFERÊNCIAS}

1. ARAMBURU GG, ARANA AL. Resolución espontánea de membrana epirretiniana idiopática en un paciente joven. Archivos de la Sociedad Española de Oftalmología, 2005; 80(12).

2. CHATZIRALLI I, et al. Treatment of macular edema after pars plana vitrectomy for idiopathic epiretinal membrane using intravitreal dexamethasone implant: long-term outcomes. Ophthalmologica, 2019; 242(1).

3. $\mathrm{CHEN} \mathrm{H}$, et al. Macular microvasculature features before and after vitrectomy in idiopathic macular epiretinal membrane: an OCT angiography analysis. Eye, 2019; 33(4).

4. GOVETTO A, et al. Functional and anatomical significance of the ectopic inner foveal layers in eyes with idiopathic epiretinal membranes: surgical results at 12 months. Retina, 2019; 39(2).

5. ISHIDA Y, et al. Risk factors, onset, and progression of epiretinal membrane after 25-gauge pars plana vitrectomy for rhegmatogenous retinal detachment. Ophthalmology Retina, 2020; 4(3).

6. JANG K, et al. Comparison of the effect of air tamponade versus no tamponade after pars plana vitrectomy for idiopathic epiretinal membrane. Scientific Reports, 2021; 11(1).

7. JUAREZ AB. Membrana Epirretiniana: caso clínico. Ciencias de la optometría: Revista trimestral de la Asociación de antiguos alumnos del Centro Boston de Optometría, 2003; 38.

8. KELLNER L, et al. 25-gauge vs 20-gauge system for pars plana vitrectomy: a prospective randomised clinical trial. British Journal of Ophthalmology, 2007; 91(7): 945-948.

9. KOKAVEC J, et al. YAG laser vitreolysis versus pars plana vitrectomy for vitreous floaters. Cochrane Database of Systematic Reviews, 2017; 6.

10. LUCENA DR. Estudo comparativo da vitrectomia posterior via pars plana associada à cirurgia de facoemulsificação, imediata ou postergada, para tratamento de membrana epirretiniana e buraco macular-Análise das alterações do segmento anterior. Dissertação. Universidade de São Paulo, 2020.

11. MARQUES MF, et al. Proliferação epirretiniana associada a buracos lamelares: implicações clínicas e cirúrgicas. Revista Sociedade Portuguesa de Oftalmologia, 2017; 41(2): 9-9.

12. NOVELLI FJ. Cirurgia da retirada da membrana epirretiniana com e sem remoção de membrana limitante interna: estudo comparativo da acuidade visual, metamorfopsia, características da tomografia de coerência óptica e taxa de recorrência. Tese de Doutorado. Universidade de São Paulo, São Paulo, 2018.

13. SÁNCHEZ-VICENTE JL, et al. Diagnóstico de enfermedad de Eales a partir de una membrana epirretiniana. Archivos de la Sociedad Española de Oftalmología, 2019; 94(11): 556-560.

14. SERRANO T. Factores pronosticos y de recuperacion de la agudeza visual tras el tratamiento de las membranas eporretinianas idopaticas. Diss. Universidad Católica de Valencia San Vicente Mártir, 2017.

15. SOUSA JJAS. Vitrectomia, um estudo computacional do procedimento cirúrgico. Dissertação submetida à Faculdade de Engenharia da Universidade do Porto - FEUP no âmbito do Mestrado Integrado em Engenharia Mecânica, 2018.

16. STAVROU P, et al. Pars plana vitrectomy in patients with intermediate uveitis. Ocular immunology and inflammation, 2001; 9(3): 141-151.

17. STEVE C, et al. Clinical comparison of 27-gauge and 23-gauge instruments on the outcomes of pars plana vitrectomy surgery for the treatment of vitreoretinal diseases. Current opinion in ophthalmology, 2020;31(3).

18. TAKAHASHI VKL. Confecção de ponteira de vitrectomia 23-gauge de dupla-porta para cirurgia de vitrectomia posterior, 2019.

19. YANNUZZI NA, et al. Internal limiting membrane peeling during pars plana vitrectomy for rhegmatogenous retinal detachment: cost analysis, review of the literature, and meta-analysis. Retina, 2018; 38(10).

20. YU Y, et al. Quantitative choriocapillaris perfusion before and after vitrectomy in idiopathic epiretinal membrane by optical coherence tomography angiography. Ophthalmic Surgery, Lasers and Imaging Retina, 2017; 48(11). 